\title{
THE MANAGER SACK RACE GAME
}

\author{
KJETIL K. HAUGEN
}

\begin{abstract}
This article applies game theory to explain why football clubs have a tendency to sack their managers when relegation is a realistic option. As opposed to coach succession literature, which typically reports contradiction between theory and practice, this article produces credible explanations for this "problem". In addition, observations indicating accelerated manager replacements in modern professional football are also easily explained. Derived Nash equilibria are also quite robust to parameter assumptions and hence the possible variations.
\end{abstract}

\section{INTRODUCTION}

Research literature related to the effects of leader replacement in business has grown considerably in the recent years. Of special interest in this article is leader replacement in sports and particularly coach replacement in association football or soccer ${ }^{1}$. This branch of research literature, often designated coach succession literature, studies possible performance changes caused by replacing managers (head coaches, field managers or trainers are different designations for this job).

According to some relevant literature surveys, [5], [1] and [6], replacement of the manager in a football team is - according to Gammelsæter in [5] - "not of much help".

Furthermore, Bridgewater [3] argues that football managers seem not only to be replaced often, but over time even more often. He based this proposed accelerating effect on data from Premier League indicating that the manager's average tenure decreased from about 3 years in 1992-1993 to 1.5 years in 2007-2008.

Without asking the obvious question, Gammelsæter concludes in [5] that there is a contradiction between theory and practice. In this author's opinion, a clearer question needs to be addressed;

If theory concludes unambiguously that replacing the manager in football has no significant positive effect, why do football clubs, and even in accelerated speed, keep on changing their managers?

Surely, one simple approach would be to hypothesize that there are serious flaws in the theory. After all, empirical before-and-after manager replacement measurements have methodological issues. Weel [9] discusses these issues in an interesting article. He claims that what one really would like to measure is the

$M S C$ (2010): primary 91A80; secondary 91A 80.

Keywords: game theory, Nash equilibrium, football manager replacement, coach succession literature.

${ }^{1}$ The term football is used in the rest of the article. 
situation where the same team plays the same matches with the old manager and the new manager simultaneously. Then, and only then, the real effect of manager replacement can be measured. Of course, the control group (playing in parallel with the old manager) is practically unfeasible. Still, he argues that many teams are in relegation battle situations, some change the manager, some do not, and it should be possible to construct some approximative control groups. Furthermore, he reports that the results are robust for different control group definitions. Unfortunately, the results do not differ much from the many empirics reported above. Admittedly, he reports significant performance improvements after replacing managers. However, similar improvements are also present in the control groups. Hence, the net effect does not contradict the existing results.

This article suggests a different path. Could it be that football clubs, and in this context their boards, do not (and should not) base their decision on replacing a manager on whether historic managerial replacements in Barcelona or Manchester City provided the desired effect? Or, could it be that such a decision should (and must) be made based on information relevant for the actual situation the club is facing? Perhaps the quality of your team and manager as well as the cost of replacement and potential effects on survival probabilities are more relevant information? Not to mention similar information related to your closest competitors, those you need to gather more points than, to avoid relegation.

These questions call for a game theoretic model. In subsequent sections and subsections, such a model is defined (Section 2), analyzed (Section 3) and discussed (Section 4). Section 5 concludes. As will be shown, such a pathway does not create contradictions between theory and practice. On the contrary, this theoretical pathway constructs a theory perfectly in line with practice.

\section{A GAME MODEL}

\subsection{Notation}

The notation used in the game model describes a situation where two professional football teams battle to avoid relegation in a league. The following notation is used:

$C_{\mathcal{C}}$ : The cost of changing (replacing) the manager in either club.

In reality, the $C_{\mathcal{C}}$-parameter will be team, league and country dependent. However, without loss of generality, a single valued (one dimensional) $C_{\mathcal{C}}$ is assumed here.

$V_{S}$ : The value of staying in the top league.

$V_{R}$ : The value of moving to a lower division - the value of relegation.

$T_{A}$ : Team A, player 1 in the game.

$T_{B}$ : Team B, player 2 in the game.

$\mathcal{K}$ : Decision - keep the manager for both teams.

$\mathcal{C}$ : Decision - change (replace) the manager for both teams.

$p_{A}^{\mathcal{K}}: \operatorname{Pr}($ Team $A$ survives the relegation battle | Keeping the manager).

$p_{B}^{\mathcal{K}}: \operatorname{Pr}$ (Team $B$ survives the relegation battle $\mid$ Keeping the manager).

$p_{A}^{\mathcal{C}}$ : $\operatorname{Pr}$ (Team $A$ survives the relegation battle | Changing the manager).

$p_{B}^{\mathcal{C}}$ : $\operatorname{Pr}($ Team $B$ survives the relegation battle $\mid$ Changing the manager). 
$\epsilon$ : A small positive real number.

$\epsilon_{A}:$ A small positive real number.

$\epsilon_{B}:$ A small positive real number.

$N$ : Number of teams in the given league.

\subsection{Assumptions}

Now, the situation at hand may be defined as follows: The two teams $T_{A}$ and $T_{B}$ have some (relatively few) games left in the season, and (for this case without loss of generality), the team ending last is relegated, while the team ending second last stays in the league. Given the relatively short time span as well as a limited number of matches left, not many other options to improve and get necessary points than changing the manager are available for the two teams ${ }^{2}$. That is, both teams face a limited set of possible decisions (in the short run) - keeping or sacking the manager. As the notation subsection above indicates, these two decisions are named $\mathcal{K}$ and $\mathcal{C}$.

So, the two teams $\left(T_{A}\right.$ and $\left.T_{B}\right)$ play a game involving choosing either $\mathcal{K}$ or $\mathcal{C}$. The information structure in the game is assumed simple. Again, given the short time horizon assumption, it seems reasonable to assume that both players make their decisions without information related to their opponent's choice. That is, we investigate a simultaneous game, a game with complete and imperfect information.

Surely, a managerial change in a football club is no secret. So, an alternative sequential formulation is feasible. However, it seems a bit too risky for one club deliberately to wait for the other club to make such a decision. Furthermore, if the other club's decision is not to change the manager, such a decision is unobservable for the opponent. Hence, the above "one-shot game" assumption seems reasonable.

Let us furthermore assume that the two teams $\left(T_{A}\right.$ and $\left.T_{B}\right)$ are located at the bottom of the league table in a league with $N$ teams. Let us also assume that, before the upcoming game is played, $T_{A}$ has the better position (located at position $N-1$ ), while $T_{B}$ holds the last place. Given this assumption, it seems very reasonable that the conditional probabilities $p_{i}^{j}, i \in\{A, B\}, j \in\{\mathcal{K}, \mathcal{C}\}$ satisfy the following inequalities:

$$
p_{A}^{\mathcal{K}}>p_{B}^{\mathcal{K}}
$$

and

$$
p_{A}^{\mathcal{C}}>p_{B}^{\mathcal{C}}
$$

In addition to the two inequalities above, two other inequalities are very reasonable to assume:

$$
p_{A}^{\mathcal{C}}>p_{A}^{\mathcal{K}}
$$

and

$$
p_{B}^{\mathcal{C}}>p_{B}^{\mathcal{K}}
$$

\footnotetext{
${ }^{2}$ In a longer time perspective, more options to improve performance is of course available - buying and selling players for instance. However, today's situation with few and relatively short transfer windows, typically not timely located at the end in the season, makes this (limited decision space for the teams) a reasonable assumption.
} 
The interpretation of inequalities (2.1) and (2.2) are simply the rationality of the players of the game. It seems very hard not accept that football teams believe that a managerial change leads to better sport performance. Nobody sacks the manager if they plan to replace him/her with a manager producing worse team performance $^{3}$.

The two values, $V_{S}$ and $V_{R}$, which are assumed to be monetary, denotes the value of staying in the league and the value of being relegated respectively. In most leagues, at least the most important ones, it seems safe to assume that $V_{S} \gg V_{R}$. See for instance [8], which reports that relegation from the Premier League may cost over $\$ 375$ million $^{4}$.

The cost of a manager change, $\left(C_{\mathcal{C}}\right)$, will also (in practice) depend a lot on the situation. Football managers, like football players, normally have a time-limited contractual obligation to a club. And, if the club needs to brake this contract, monetary compensation will normally be necessary. Dupare [4] tells some stories about especially costly managerial changes in Premier League football clubs (with replacement costs ranging from $£ 11$ million to $£ 18$ million), as such perhaps not representing average costs. Still, although these numbers are high, they are of different magnitudes than relegation costs. As a consequence, it is reasonable to assume that $C_{\mathcal{C}} \ll V_{S}, C_{\mathcal{C}} \ll V_{R}$ as well as $C_{\mathcal{C}} \ll V_{S}-V_{R}$.

In order to complete the game formulation, risk neutral teams and expected value maximization as objectives for both club are assumed.

\subsection{Modeling}

Given all assumptions made in Subsection 2.2, the normal form game in Figure 1 is easily formulated.

As can be observed in Figure 1, the players' pay-off's are simply calculated as expected values of being relegated or staying in the league with additional subtracted costs, $C_{\mathcal{C}}$, if the team has chosen to change manager.

\section{Analyzing the game model}

\subsection{The $\{\mathcal{C}, \mathcal{C}\}$ Nash Equilibrium}

Analyzing the game (examining Nash Equilibria (NEs)) of Figure 1 is straightforward. The most interesting outcome here, existence of the $\{\mathcal{C}, \mathcal{C}\}$ NE, is examined first.

To secure that the $\left\{C_{\mathcal{C}}, C_{\mathcal{C}}\right\}$-outcome is a $\mathrm{NE}$, the following two inequalities must be satisfied:

$$
p_{A}^{\mathcal{C}} V_{S}+\left(1-p_{A}^{\mathcal{C}}\right) V_{R}-C_{\mathcal{C}}>p_{A}^{\mathcal{K}} V_{S}+\left(1-p_{A}^{\mathcal{K}}\right) V_{R}
$$

and

$$
p_{B}^{\mathcal{C}} V_{S}+\left(1-p_{B}^{\mathcal{C}}\right) V_{R}-C_{\mathcal{C}}>p_{B}^{\mathcal{K}} V_{S}+\left(1-p_{B}^{\mathcal{K}}\right) V_{R}
$$

\footnotetext{
${ }^{3}$ There could of course be special (and quite improbable) situations where the existing manager is too expensive for today's budget. That is, you replace the manager due to manager costs, but such sitations are very rare, and not intended to be of relevance in this gaming situation.

${ }^{4}$ Obviously, such estimates are both uncertain and very simplified. Additionally, the club, league and country has a great impact on actual numbers. Still, in most situations, relegation in football is costly, or as we state it here: $V_{S} \gg V_{R}$.
} 


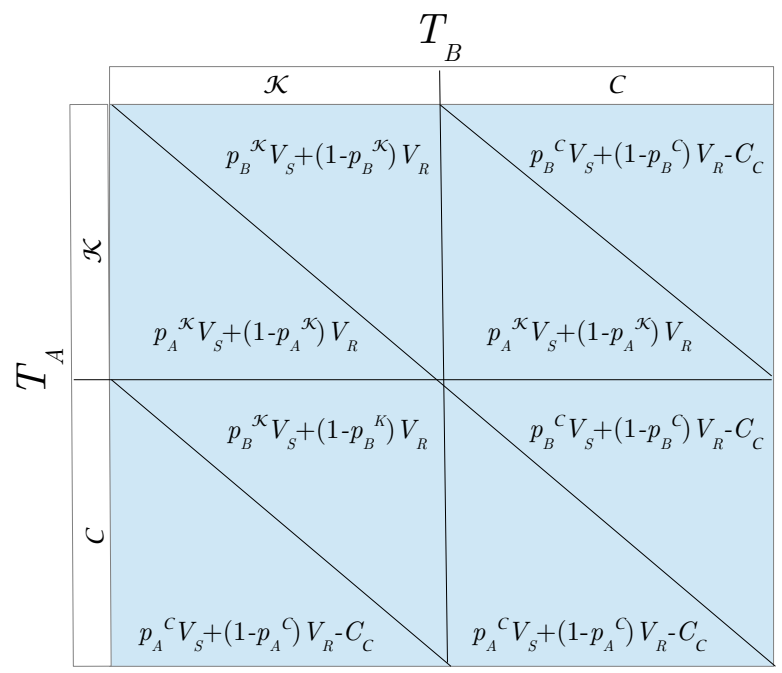

Figure 1. A normal form game formulation.

Applying assumption (2.1) we find:

$$
p_{A}^{\mathcal{C}}>p_{A}^{\mathcal{K}} \Rightarrow p_{A}^{\mathcal{K}}+\epsilon_{A}=p_{B}^{\mathcal{C}} .
$$

Now, substituting this value of $p_{A}^{\mathcal{C}}$ into inequality (3.1) we find:

$$
\left(p_{A}^{\mathcal{K}}+\epsilon_{A}\right) V_{S}+\left(1-\left(p_{A}^{\mathcal{K}}+\epsilon_{A}\right)\right) V_{R}-C_{\mathcal{C}}>p_{A}^{\mathcal{K}} V_{S}+\left(1-p_{A}^{\mathcal{K}}\right) V_{R} .
$$

Some simple algebra gives:

$$
p_{A}^{\mathcal{K}} V_{S}+\epsilon_{A} V_{S}+V_{R}-p_{A}^{\mathcal{K}} V_{R}-\epsilon_{A} V_{R}-C_{\mathcal{C}}>p_{A}^{\mathcal{K}} V_{S}+V_{R}-p_{A}^{\mathcal{K}} V_{R}
$$

and

$$
\epsilon_{A} V_{S}-\epsilon_{A} V_{R}-C_{\mathcal{C}}>0 \text { or } \epsilon_{A}>\frac{C_{\mathcal{C}}}{V_{S}-V_{R}} .
$$

Now, a completely similar derivation can be performed for $T_{B}$ - defining $p_{B}^{\mathcal{C}}>$ $p_{B}^{\mathcal{K}} \Rightarrow p_{B}^{\mathcal{K}}+\epsilon_{B}=p_{B}^{\mathcal{C}}$ and substitution into inequality $(3.2)-$ to produce $\epsilon_{B}>$ $\frac{C_{\mathcal{C}}}{V_{S}-V_{R}}$. At this point, it is convenient to simplify ${ }^{5}$ and assume that $\epsilon_{A}=\epsilon_{B}=\epsilon$ and as a consequence end up with:

$$
\epsilon>\frac{C_{\mathcal{C}}}{V_{S}-V_{R}}
$$

to secure the $\{\mathcal{C}, \mathcal{C}\}$ NE.

${ }^{5}$ This simplification is made to obtain a single expression for the inequality defining the NE. Furthermore, the actual meaning of the $\epsilon$-term, which is the probabilistic gain of changing the manager to stay in the league, is probably almost the same for the two fairly bad teams anyway, located at the very bottom of the table as they are. 


\subsection{Uniqueness of the $\{\mathcal{C}, \mathcal{C}\}$ NE}

In the previous subsection (Subsection (3.1)), it was shown that the outcome $\{\mathcal{C}, \mathcal{C}\}$ is a NE. Or, in equilibrium, both teams choose to replace their manager. However, the possible uniqueness was not proven. In this subsection, we will investigate the possible uniqueness properties of this $\mathrm{NE}^{6}$.

Assume now that inequality (3.3) is satisfied. Given this assumption, it is straightforward to realize that the outcomes $\{\mathcal{C}, \mathcal{K}\}$ and $\{\mathcal{K}, \mathcal{C}\}$ are impossible as candidates for NE-status. This is easy to see by realizing that should $\{\mathcal{K}, \mathcal{C}\}$ be a NE-candidate, the following inequality would have to be satisfied:

$$
p_{A}^{\mathcal{C}} V_{S}+\left(1-p_{A}^{\mathcal{C}}\right) V_{R}-C_{\mathcal{C}}<p_{A}^{\mathcal{K}} V_{S}+\left(1-p_{A}^{\mathcal{K}}\right) V_{R}
$$

Similarly, for $\{\mathcal{C}, \mathcal{K}\}$ to be a NE-candidate,

$$
p_{B}^{\mathcal{C}} V_{S}+\left(1-p_{A}^{\mathcal{C}}\right) V_{R}-C_{\mathcal{C}}<p_{B}^{\mathcal{K}} V_{S}+\left(1-p_{K}^{\mathcal{K}}\right) V_{R}
$$

Comparing inequalities (3.4), (3.5) with (3.1), (3.2) it is readily observed that the pairs are equal apart from the inequality sign. Then, given the assumption of a satisfied (3.3), neither (3.4) nor (3.5) can be satisfied, and hence, neither $\{\mathcal{C}, \mathcal{K}\}$ nor $\{\mathcal{K}, \mathcal{C}\}$ are NEs in the game.

Then, the only remaining possible (pure strategy) NE candidate is $\{\mathcal{K}, \mathcal{K}\}$. Again, given the assumption of (3.3), we are looking at a potential situation with 2 pure $\mathrm{NEs}^{7}$, and as most standard text books in Game Theory ${ }^{8}$ might name a "Stag-Hunt" game. For this to happen, the following two inequalities must be satisfied:

$$
p_{A}^{\mathcal{C}} V_{S}+\left(1-p_{A}^{\mathcal{C}}\right) V_{R}-C_{\mathcal{C}}<p_{A}^{\mathcal{K}} V_{S}+\left(1-p_{A}^{\mathcal{K}}\right) V_{R}
$$

and

$$
p_{B}^{\mathcal{C}} V_{S}+\left(1-p_{A}^{\mathcal{C}}\right) V_{R}-C_{\mathcal{C}}<p_{B}^{\mathcal{K}} V_{S}+\left(1-p_{K}^{\mathcal{K}}\right) V_{R}
$$

We observe readily that $(3.6)=(3.4)$ and $(3.7)=(3.5)$. As a consequence, the "Stag-Hunt" game is impossible in this game, and we can conclude that the game has two potential unique pure NEs.

$$
\text { If } \epsilon>\frac{C_{\mathcal{C}}}{V_{S}-V_{R}} \text { the game has the unique pure strategy NE: }\{\mathcal{C}, \mathcal{C}\}
$$

or (by simple logic):

$$
\text { If } \epsilon<\frac{C_{\mathcal{C}}}{V_{S}-V_{R}} \text { the game has the unique pure strategy NE: }\{\mathcal{K}, \mathcal{K}\} .
$$

That is, the game produces only two alternative NE's. Either both teams keep, or both replace their manager. This particularly simple game prediction will prove relevant for the discussion in Section 4.

\footnotetext{
${ }^{6}$ In the argument following, it is assumed that possible equalities between various pay-off expressions in Figure 1 are non-existent. In practice the probabilities of such equalities are certainly very low, so this seems to be a fair assumption.

${ }^{7}$ This case also contains a third NE in mixed strategies. This is, however, of no importance here.

${ }^{8}$ See for instance [2].
} 


\section{Discussion}

\subsection{The probable NE}

At this point, it will prove convenient to return to a discussion on the potential practical values of the parameters in inequality (3.3), determining the NE.

First, the value of relegation (defined as $V_{S}-V_{R}$ here) was briefly discussed in Subsection 2.2. There, the value of relegation from Premier League was reported to be at least $\$ 375$ million (or around $£ 315$ million in today's currency) for the upcoming 20/21 season. This estimate was based on a statement by an Aston Villa executive. Clearly, this is a highly uncertain estimate and perhaps strongly linked to the actual club and its position at the time. Still, as it is based on Premier League prize money as well as TV-revenue, it defines the type of club. This is not a top 5 or 6 club that does not fight relegation, but fighting to enter the European scene - Champions or Europa League. Such a club will most certainly have far higher costs of relegation. Surely, the value of competing in the top level European tournaments is also highly dependent on the actual team and the country it comes from, but some numbers are transparent and available. According to [7], the upcoming season's (20/21) winner of CL would receive a cash-flow of $£ 74$ million if all group stage matches were won. In addition, a certain share of the so-called "Market Pool" money will also be added. For the upcoming season this is estimated to be $£ 263$ million. In addition to this, added sponsor money, shirt sales etc. will most certainly also be lost if (say) Manchester United faces relegation to the championship.

The point is simple. If a better club (not Aston Villa) faces relegation, the cost of relegation will be significantly higher than the estimate of $£ 315$ million discussed above. A total cost of (say) $£ 500$ million does not seem unreasonable.

The point of this being practical, is to try to judge the magnitude of the righthand side of inequality (3.3). The reason for re-estimating the "Aston Villa" to a "Manchester United" estimate is to be able to compare with the relevant information on the denominator in the fraction, $\frac{C_{\mathcal{C}}}{V_{S}-V_{R}}$. Dupare [4] lists the 5 most expensive manager switches in Premier League history. They range from $£ 11$ to $£ 18$ million. Given that these values may be seen, at least as an upper bound on a managerial replacement today, we have comparable numbers. Say, for instance, that two clubs in our game formulation expect that they (at worst) may need $£ 15$ million $^{9}$ to replace their managers, the fraction can be computed as: $\frac{C_{\mathcal{C}}}{V_{S}-V_{R}}=\frac{15}{500}=0.03$ or more conveniently; $3 \%$.

This means that in order to satisfy inequality $3.3, \epsilon>0.03$ or in more practical terms; if you are a member of the board in Manchester United, atypically struggling to stay in Premier League, you are facing the following decision: If we can replace our manager with a new one that increases the probability of staying in the league by merely $3 \%$, we should do it. Such a decision seems easy to make, in a situation where your beloved club literally stands in front of the cliff.

Surely, the argumentation above is highly uncertain and involves too many practical short cuts. Still, the fact that the cost of relegation must be much higher than the managerial replacement must be correct. After all, we constantly observe

\footnotetext{
${ }^{9}$ Based on a simple average estimate: $\frac{11+18}{2}=14.5 \approx 15$.
} 
that football clubs change their managers. For instance, this season in Norway (2020) , before half of the matches have been played, 3 out of 16 team mangers have already been sacked.

That is, it does seem very probable to expect that football teams, given a reasonable belief that the formulated model reasonably represents reality, quite often will replace their managers. It is not a stupid decision after all. It is on the contrary a very rational decision, even if all empirical information in the world tells them that it does not help.

The above arguments were based on a case from UK Premier League. What about other leagues in other countries? Of course, the numbers may and will change. In less valuable leagues, these numbers are definitely different, and typically significantly smaller. However, the point is not the absolute values of the

numbers, but the fact that $V_{S}-V_{R} \gg C_{\mathcal{C}}$ and consequently, $\frac{C_{\mathcal{C}}}{V_{S}-V_{R}}$ is a small number. This seems to be the case in most other countries other than UK, and this model may prove an interesting explanation why football teams tend to change their managers when performance problems occur globally.

Surely, we have modeled a situation involving two teams struggling to avoid relegation. It is important to be aware of the fact that football teams may have other and very good other reasons to change managers. Such situations, involving for instance end of contracts, health issues, age, general performance issues, salary costs and so on are not explicitly modeled here. That is, the main focus here, is a situation where relegation is a probable outcome.

\subsection{Game model discussion and critique}

As the previous section indicates, the validity of the arguments and the resulting $\mathrm{NE}$ is perhaps more focused on the actual game model and its assumptions as opposed to the actual numbers deciding the Nash equilibrium.

One obvious simplification in the model is the number of teams and, hence, players. Surely, the choice of two teams instead of three or four is not random. After all, in the most practical leagues, more than one team is relegated; typically 2-4 depending on some play-off structure. As such, the choice is made to simplify the mathematical analysis, but similar modeling and arguments could still be performed for two and two teams at a time, and is seems very unlikely that the results should change dramatically.

The fact that we simplified by setting $\epsilon_{A}=\epsilon_{B}=\epsilon$ in Subsection 3.1, may (typically) complicate the structure of NEs in the game. It may for instance open up for "Chicken-type" situations, that is non-coordinating solutions with potential NEs such as $\{\mathcal{C}, \mathcal{K}\}$ or $\{\mathcal{K}, \mathcal{C}\}$. Still, as briefly discussed previously (also in Subsection 3.1), the probability that teams struggling with each other to avoid relegation are quite equal in quality and wealth (or other possible relevant dimensions) is quite high. After all, Manchester United and Liverpool are quite seldom facing the relegation option.

The choice of a simultaneous (imperfect information) game can of course also be discussed. It was done briefly in Subsection 2.2. The point that the $\mathcal{K}$ decision is unobservable before the league ends is a good argument for not choosing a different information structure in the game formulation. Still, a situation where one team 
suspects that the other team may have plans to replace their manager (just like we have) is relevant and definitely interesting. However, such an assumption would lead to far more complex formulations involving for instance espionage decisions.

In reality, most actual practical games will contain elements calling for games of incomplete information. Here, the game formulation in Figure 1 implicitly assumes that all information in Figure 1 is available for both players ${ }^{10}$. In the real world one could perhaps expect that $T_{A}$ should have information about (or at least principally information available to estimate) $P_{A}^{\mathcal{K}}, P_{A}^{\mathcal{C}}$, but having the same type of information on your closest opponent, in this case, $T_{B}$ may be harder to accept. As such, a game model of incomplete and possibly asymmetric information seems clearly relevant. This a very different approach and was not the intention of this article - still, clearly of interest.

However, intuitively, if you do not know your opponent, it seems reasonable to expect even more tendency to fire the $\operatorname{coach}^{11}$ and, hence, even stronger results than the complete information version presented here.

\subsection{NE structure}

The obvious simplicity of the game solution, as indicated by the inequalities (3.8) and (3.9) in Subsection 3.2, is interesting. The fact that our game produces a single pure unique NE tells a history of predictive power. That is, a game with such a solution structure indicates a higher degree of trust in the solution than alternative outcomes - "Stag-Hunt" or "Chicken games" for instance.

Actually, the situation provides an even stronger predictive power. In Figure 2, the best reply functions for both players $T_{A}$ and $T_{B}$ are shown with (red) ellipses and rectangles for players $T_{A}$ and $T_{B}$, respectively. These best reply functions are calculated under the assumption that inequalities (3.1) and (3.2) (leading to (3.3)) are satisfied.

As can be observed from the best reply functions in Figure 2, both players play dominant strategies in this game. That is, no matter what decision your opponent takes, you will always choose to change your manager - the $\mathcal{C}$ option. This is often referred to as a Nash equilibrium in dominant strategies. A NE in dominant strategies is (logically) a subset of NEs with especially strong predictive power. After all, if the player you play against, always does the same, no matter what choice you make, it is easy to find your choice, and the predictive power in such situations is especially strong.

To sum up, given that the formulation is accepted, the game solution provides the strongest possible predictive power in game theory. Or in more popular terms, it is (at least) no doubt related to the game solution. It should be a very clear indication of what rational players would and should do in the game.

\footnotetext{
${ }^{10}$ This is a consequence of the choice of a complete information game formulation,

${ }^{11}$ The actual NE contingency consequences will of course depend on the information assumptions on opponent types. But if one assumes some kind of symmetry on the quality of the teams, such an outcome seems probable. Of course to sort out these things scientifically, an incomplete game model must be formulated and analyzed, clearly beyond the scope of this article.
} 


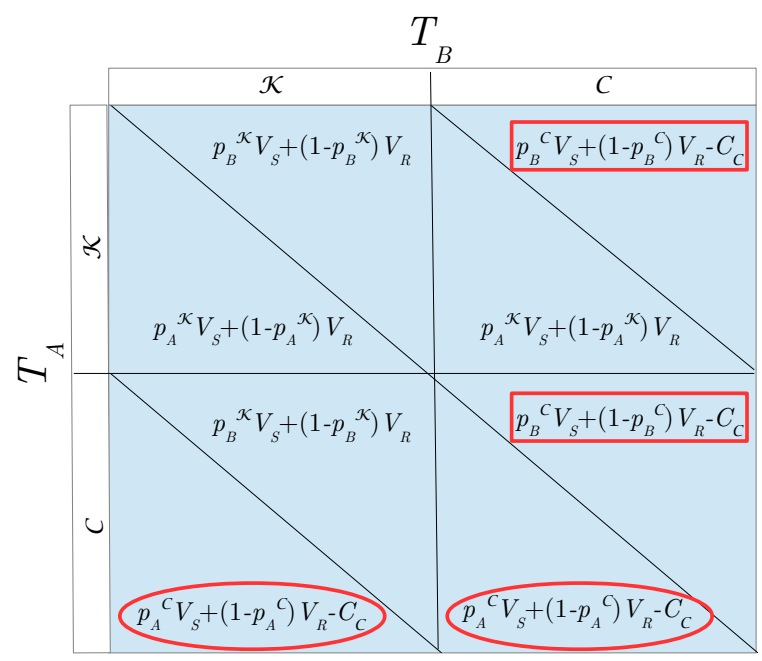

Figure 2. Best reply functions.

\section{Conclusions}

This article has demonstrated that a game theoretic model may produce coherence between theory and practice in football coach succession. The model shows by some rough estimates of relevant parameters that the probable (and rational) decision for a football club in serious trouble is to replace their manager. That is, football boards are not necessarily fools, as alternative theory may indicate. On the contrary, they are perfectly rational, given that the model assumptions used are acceptable.

Furthermore, indications of acceleration effects (i.e. modern football consumes managers even faster than before) can be easily accounted for as the relegation costs are imperative in establishing NEs. The fact that the relegation costs are decisive in securing the essential model result (see inequality (3.3) and that high relegation costs secure the direction of the inequality, means that if modern football produces higher relegation costs, more manager replacements is a definitive result.

Finally, the results are robust in the sense that NEs are unique and pure. In addition, the NEs are of a dominant strategy type, which should make them even easier to believe being the actual game prediction.

\section{REFERENCES}

[1] J. A. Andersen, A new coach does not make a better team, Int. J. Sports Sci. Coach. 8 (2011), 167-178.

[2] K. Binmore, Fun and Games: A Text on Game Theory, D. C. Heath and Company, Lexington, MA, 1992.

[3] S. Bridgewater, Football Management, Palgrave Macmillan, Basingstoke, Hampshire, New York, NY, 2010.

[4] S. Dupare, 5 of the biggest compensation packages ever paid to football managers, https://www.sportskeeda.com/football/5-of-the-biggest-compensation-packages-everpaid-to-football-managers/5, Sportskeeda, December 2018. 
[5] H. Gammelsæter, Leader succession and effectiveness in team sport, a critical review of the coach succession literature, Sports, Bus. Manag. 3 (2013), 285-293.

[6] R. C. Giambatista, W. G. Rowe and S. Riaz, Nothing succeeds like succession: A critical review of leader succession literature since 1994, Leadersh. Q. 16 (2005), 963-991.

[7] Goal, How much money do the champions league 2019-20 winners get? https://www.goal.com/en/news/how-much-money-champions-league-2019-20-winnersget/1b78sfes1al5k1cxve9ld1wp48, August 2020.

[8] R. Harris, Over \$375 million at stake for teams fighting relegation in EPL finale, https://www.stuff.co.nz/sport/football/world-game/300066053/over-375-million-atstake-for-teams-fighting-relegation-in-epl-finale, Stuff, July 2020.

[9] B. ter Weel, Does manager turnover improve firm performance? Evidence from Dutch soccer, 1986-2004, De Economics 159 (2011), 279-303.

Kjetil K. Haugen, Faculty of Business Administration and Social Sciences, Molde University College, Specialized University in Logistics, Molde, Norway

e-mail: kjetil.haugen@himolde.no 
